\title{
Source of the catalytic activity of gold nanoparticles
}

\author{
Geoffrey Bond \\ www.goldbulletin.org
}

\begin{abstract}
Analysis of literature results for the effect of particle size in $\mathrm{Au} / \mathrm{TiO}_{2}$ catalysts on their activities and activation energies (E) for CO oxidation by constructing 'Compensation' plots of $E$ vs. In $A$ ( $A$ = pre-exponential factor) leads directly to the conclusion that high activity is primarily due to particles so small as to be in the non-metallic state.
\end{abstract}

\section{Introduction}

Towards the end of 2008, David Thompson and I began a survey of the literature on the performance of gold catalysts, particularly for $\mathrm{CO}$ oxidation, in preparation for submitting an abstract to GOLD2009. We compiled a long list of reported rates of this reaction for many types of catalyst, our aim being to identify those features of composition, structure and pre-treatment that affected activity and stability, either in storage or in use. We were of course aware of the importance of gold particle size, of type of support, and of certain poisons such as $\mathrm{Cl}^{-}$, but with one or two notable exceptions we could not find any systematic or quantitative studies of the effects of these or the many other likely variables. The scheme which we constructed to summarise them, and which appeared as Scheme 1 in the recent publication (1) based on the GOLD2009 abstract, was therefore based on numerous qualitative or semi-quantitative observations in the literature.

A particular difficulty that we encountered in analysing the literature data was the widely differing conditions under which rates were measured. These included importantly temperature, the $\mathrm{O}_{2} / \mathrm{CO}$ ratio, and the time-on-stream (which was not always specified); rates had often to be calculated from values of $T_{50}$. To deal with the temperature variation, we plotted rates for a given type of catalyst on Arrhenius diagrams (i.e. In rate vs. 1/T), and set lines through them corresponding to an activation energy of $30 \mathrm{~kJ} \mathrm{~mol}^{-1}$, which is a typical value. We were surprised to find how widely scattered our data points were on these diagrams; although it was possible to detect a zone in which many of the more active catalysts were located, many lay below it in a seemingly random scattering of points. A few lay well above this zone, due to superior experimental technique, optimism or computational error. For a single catalyst, $\mathrm{Au} / \mathrm{TiO}_{2}$, the plot contained more 
than 60 data points; the rates at $298 \mathrm{~K}$ differed by a factor of over 100. Plots of this type, made for other types of catalyst, behaved similarly; they are not worth showing, as it is clearly impossible to extract useful information from them.

Another approach had therefore to be tried, and attention was focused on one of the few papers in which a single parameter of a catalyst, namely, the particle size of the gold, had been systematically altered and its effect on $\mathrm{CO}$ oxidation determined (2). In fact two catalysts containing different gold loadings (4.5 and $7.2 \%$ ) were studied, and each was calcined at a series of temperatures up to 773 $\mathrm{K}$; particle sizes were estimated by XAFS, using a truncated cubo-octahedral model. The rates afforded by the twelve catalysts were measured over a range of temperatures, sometimes in duplicate, and activation energies and rates at 273 and $298 \mathrm{~K}$ were tabulated. Selected Arrhenius plots were shown, but standard deviations on activation energies were not given. It is worth stressing that in datasets such as this, where the activation energy is variable, there is no temperature at which a unique profile of rate vs. particle size can be obtained. Two kinds of rate were reported; the first, named the atomic rate, is the rate expressed per total mass of metal used $\left(\mathrm{mol} \mathrm{CO}\left(\mathrm{mol} \mathrm{Au}_{\mathrm{tot}} \mathrm{s}\right)^{-1}\right)$, and the second is a pure turnover frequency (mol CO $\left.\left(\mathrm{mol} \mathrm{Au} u_{\text {surf }} \mathrm{s}\right)^{-1}\right)$, which is the observed rate corrected for the variation of mean particle size.

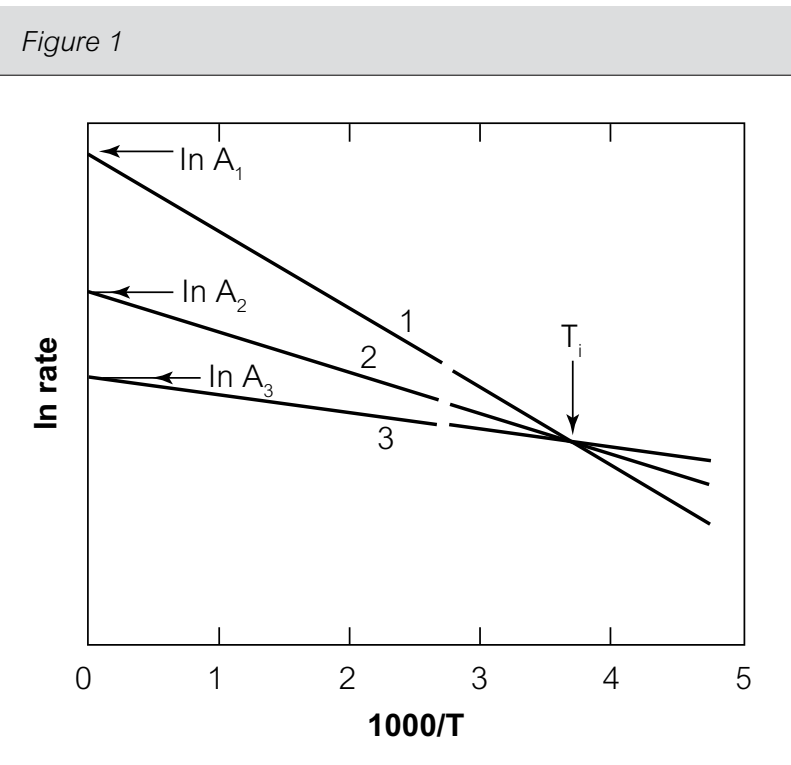

Three Arrhenius plots (In rate vs. 1/T) showing 'Compensation' and intersecting at the isokinetic point $T_{i}$; the activation energies are proportional to the slopes $(E=s \times R)$, and the In A terms are the intercepts at $1 / T=0$

\section{The Compensation phenomenon}

It is now necessary to introduce the idea of 'Compensation' in heterogeneous catalysis. The effect of temperature on rate may be expressed by the Arrhenius Equation in the form

rate $=A_{\text {app }} \exp \left(-E_{\text {app }} / R T\right)$

where $E_{\text {app }}$ is the apparent activation energy, uncorrected for any change in the concentration of adsorbed reactants over the temperature range employed, and $A_{\text {app }}$ is a portmanteau term that includes the number of active sites and the entropy of activation (3). It has the same dimensions as the rate, and corresponds to the value of the rate when $1 / T$ is zero; it is simply evaluated from the Arrhenius Equation expressed as

In rate $=\ln \mathrm{A}_{\mathrm{app}}-\mathrm{E}_{\mathrm{app}} / \mathrm{RT}$.

All that is needed is a value for $E_{\text {app }}$ and the rate at a specified temperature.

Now it is very often observed that when this information is available for a number of catalysts and a single reaction, or for a number of related reactions on a single catalyst, that there is a linear relation between $\ln A_{\text {app }}$ and $E_{\text {app }}$;

$\ln \mathrm{A}_{\mathrm{app}}=m \mathrm{E}_{\mathrm{app}}+\mathrm{C}$.

When this happens, a high $E_{\text {app, }}$ which normally betokens a low rate because of the exponential term, is compensated by a high In $A_{\text {app. }}$. For this relation to hold exactly, the individual Arrhenius plots in the dataset must all intersect at precisely the same point, termed the isokinetic point, and at higher temperatures the member having the highest $E_{a p p}$ is the most active, the opposite being true at lower temperatures (Figure 1). The literature reveals many examples of this behaviour (3-5), and its cause has been much debated, but it now appears (5) that it arises from the use of apparent Arrhenius parameters, and not the true parameters that need to be extracted from the temperature-dependence of the rate constant $k$, i.e.

rate $=k P_{A}{ }^{a} P_{B}{ }^{b}$

where $a$ and $b$ are respectively the 'orders of reaction' for the two reactants $\mathrm{A}$ and $\mathrm{B}, P$ being their pressure. 


\section{Use of the compensation concept in CO oxidation}

It was therefore natural that we should seek to test the results mentioned above (2), to see if they demonstrated 'Compensation'. For the first test, the pure TOF values were used to compute values of In $A_{\text {app }}\left(A_{\text {app }}\right.$ then has the same dimensions as TOF); the resulting plot (Figure 2) contains 19 points, as duplicate values of the rate were cited for many of the drying/calcination temperatures. Note that in this and later figures $A_{\text {app }}$ is expressed

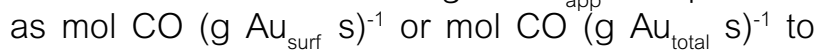
facilitate comparison with other work. Values of In $A_{\text {app }}$ calculated from each of the two temperatures at which rates were quoted were essentially the same. The mean particle size for each point is indicated. Although there is a general sense of 'Compensation', the points fall in no particular order, and the linearity of the plot is much inferior to many in the literature (3-5). The implication is that rates do not depend on the surface areas of the particles. This is perhaps not a surprising conclusion, in view of the stress that has been placed on edge and corner atoms, and on peripheral sites.

However, a plot based on values of In $A_{a p p}$ derived from the atomic rates is very different and much

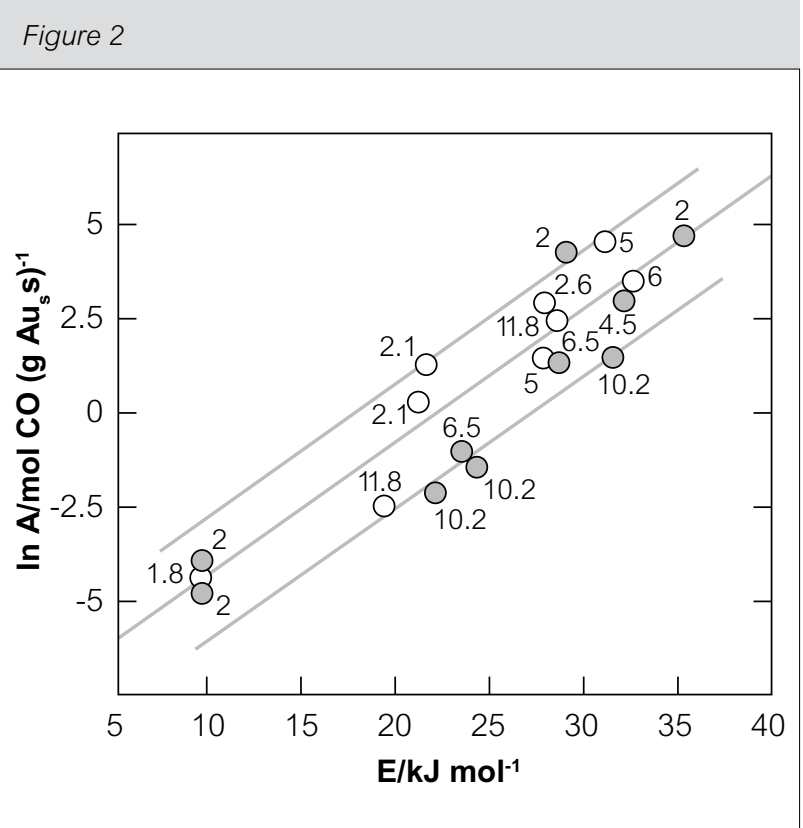

'Compensation' plot of $E_{a p p}$ vs. In $A_{\text {app }}$ for CO oxidation on $\mathrm{Au} / \mathrm{TiO}$, catalysts having various particle sizes as indicated in $\mathrm{nm}$ (2), $A_{\text {app }}$ being calculated from rates corrected for differences in surface areas (units, $\mathrm{mol} C \mathrm{CO}\left(\mathrm{mol} \mathrm{Au}_{\text {surf }} s\right)^{-1}$. Open points, 4.5\% Au; hatched points. 7.2\% Au more informative (Figure 3); in this Figure $A_{\text {app }}$ has the units of mol $\mathrm{CO}\left(\mathrm{g} A u_{\text {total }} \mathrm{s}\right)^{-1}$, the rate thus expressed being the atomic rate divided by the atomic mass of gold. In this case, all eight points for samples having particles of mean size less than $3 \mathrm{~nm}$ fall about a single line, while those having larger particles fall around another line of steeper slope, their rates being naturally lower than those of the other set. In neither case is the linearity perfect, and the points for the $5 \mathrm{~nm}$ particle size tend to adopt intermediate positions, but the separation of the two groups in this way is unmistakable and highly significant. Thus rates based on the average mass of gold in each particle fall neatly into two groups, showing respectively high and low activities.

Before examining further the implications of this divergence, it is worth looking to see if it is supported by results on other catalysts. Unfortunately the evaluation of activation energy is not always performed, even when the dependence of conversion on temperature is reported; as a referee, I frequently deplore this. It is important to squeeze every last drop of information from costly experimental work. Nevertheless there are a number of values of activation energy in the literature, especially in publications based on the work of F. Moreau (6-9), perhaps due to the influence of his adviser. Figure 4 shows a plot of In $A_{a p p} v s$. $E_{a p p}$ for some of these additional results, which cover gold catalysts supported on $\mathrm{TiO}_{2}, \mathrm{Al}_{2} \mathrm{O}_{3}$, and other single

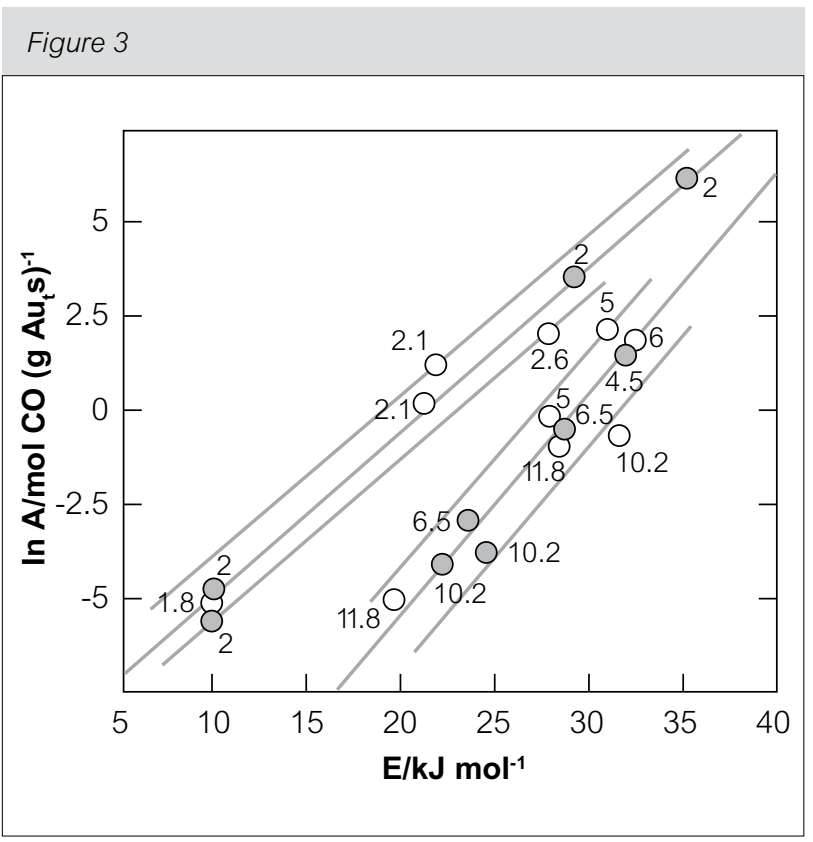

The equivalent plot to Figure 2, but with $A_{a p p}$ calculated from rates expressed as $\mathrm{mol} \mathrm{CO}\left(\mathrm{mol} A u_{\text {total }}^{\text {app }}\right)^{-1}$ 


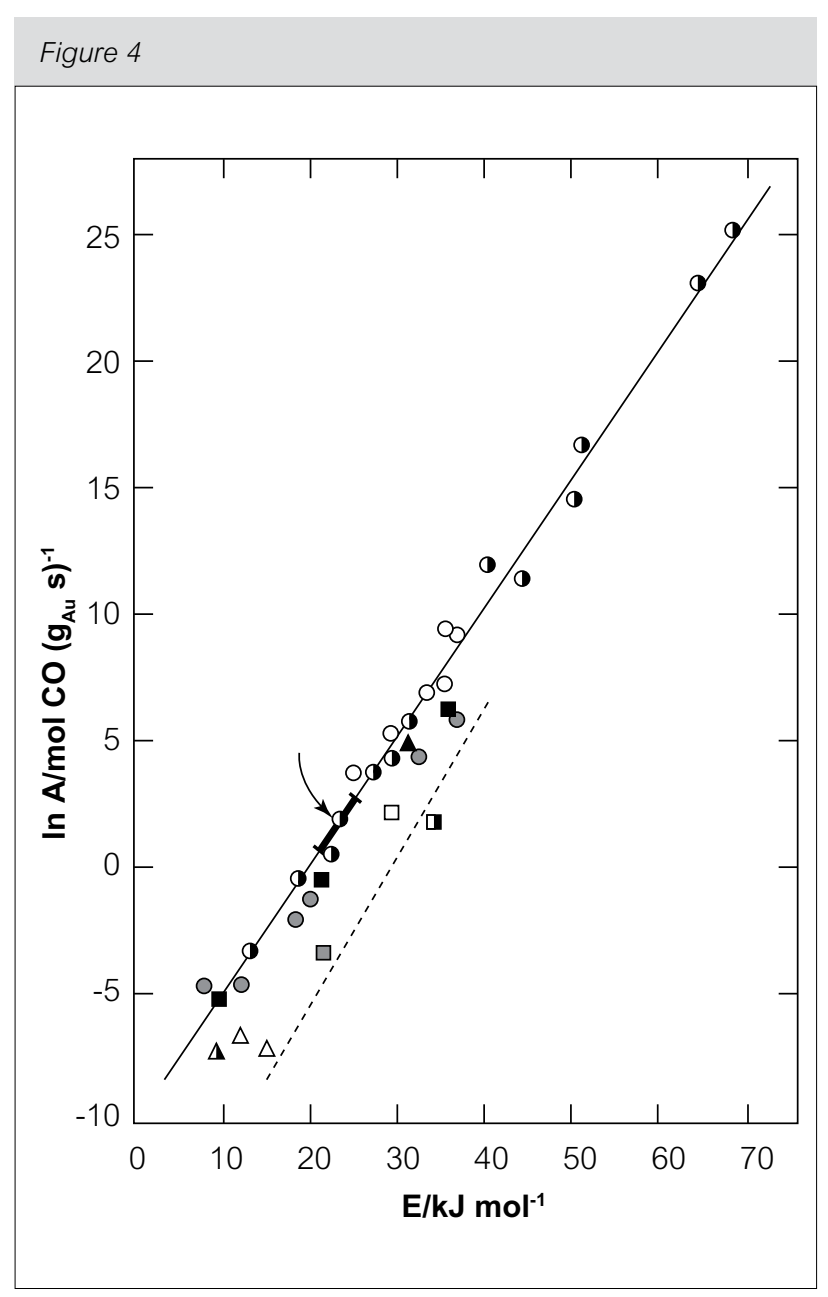

A 'Compensation' plot for CO oxidation on various gold catalysts; the broken line is equivalent to the lower line in Figure 3. The supports are: open points, $\mathrm{TiO}_{2}$; hatched points, $\mathrm{Al}_{2} \mathrm{O}_{3}$; half-filled points, other supports. Other symbols: $\mathbf{\Delta}$ World Gold Council AulTiO ; points from Figure 3 for small particle catalysts; other squares, catalysts made by cluster beam deposition (14); $\triangle$ catalysts with large gold particles (10). Other points are derived from references 6-9 and J. Catal. 2001, 113, 197; J. Catal. 2006, 238, 458; Catal. Lett.1998, 51, 53; and Catal. Lett. 2005, 99, 21

and binary oxides. Many of the points lie close to a line that covers a range of $E_{a p p}$ of 10 to $70 \mathrm{~kJ}$ $\mathrm{mol}^{-1}$; many of the values for $\mathrm{Au} / \mathrm{TiO}_{2}$ lie in the zone 25-35 kJ mol-1, and not all such results are included in this plot. Also shown in the Figure are three of the points from Figure 3 for the catalysts having small particles; they also lie close to the line through the majority of the points. Although in some cases the metal particle size in the other catalysts shown in this Figure were not measured, from their generally high activity it may be inferred that most were below about $3 \mathrm{~nm}$. This long line may therefore be taken as characteristic of the nanoparticulate state.
The lower line drawn in Figure 3 through the points for catalysts having larger particles is repeated in Figure 4, together with three points for catalysts with particles of 12-18 nm size (10) (triangles). These appear to be more active than those made by heat treatment (2) on which the line in Figure 3 was based; this suggests that their low activity may not have been solely due to their larger size, as heating may have produced other relevant changes, such as dehydroxylation of the support, which diminish activity. However the consensus of much work is that particle size is of vital importance, and that it rises abruptly below about $3 \mathrm{~nm}$, irrespective of the support (11). High activities have been reported for very small gold particles on $\mathrm{Al}_{2} \mathrm{O}_{3}(1)$, which is less easily dehydroxylated than $\mathrm{TiO}_{2}$; it would be of interest to see Overbury's work (2) repeated with $\mathrm{Al}_{2} \mathrm{O}_{3}$ as support. Three points are shown for catalysts made by cluster beam deposition (12) (squares); these also lie about the lower line, even although their particle size is only $2.6-2.9 \mathrm{~nm}$. This implies that small size by itself is not enough; effective cooperation with the support is also needed to secure best activity. It seems to be extraordinarily difficult to find a way of altering one aspect of a catalyst's structure, such as its particle size, without simultaneously altering one or more other parameters that may bear on its activity.

In Figure 3, the rates at $273 \mathrm{~K}$ along the upper line at $E_{\text {app }}$ values of 10 and $70 \mathrm{~kJ} \mathrm{~mol}^{-1}$ cover a range of about 100; the difference in rates between the upper and lower lines at $\mathrm{E}_{\text {app }}=30 \mathrm{~kJ} \mathrm{~mol}^{-1}$ is about a factor of 200. The isokinetic temperature $T_{i}$, i.e. the temperature at which all the Arrhenius plots should intersect, is given by

$S=\left(R T_{i}\right)^{-1}$

where $s$ is the slope of the line; for the upper line $\mathrm{T}_{\mathrm{i}}$ is $227 \mathrm{~K}$, so this means that most of the results will have been collected above this temperature. Higher activities are therefore found at higher value of $E_{\text {app }}$ (see Figure 1). Some caution needs to be exercised in assigning significance to 'Compensation' plots, as the accuracy with which $E_{\text {app }}$ values are measured is often not stated. An error of $+/-10 \%$ is not unlikely in most cases, and this produces a corresponding uncertainty in In A; the effect of this is shown in Figure 3 for a single indicated point. It is inevitable that not all points should conform to a linear 'Compensation' line, as the value of In A comes directly from a measured rate, which is in turn influenced by inter alia the reactant pressures used, 
and this can sometimes make a great difference; however an error of $+/-10 \%$ in the rate makes very little difference to the value of In $A_{a p p}$. The well-known tendency of gold catalysts to suffer activity loss during CO oxidation, and lack of information concerning the time at which the rate was measured, will also cause points to wander from their line. However, notwithstanding these uncertainties, the range of $\mathrm{E}_{\mathrm{app}}$ values covered in the literature, and shown in Figure 3, together with the degree of adherence of many points to the upper line, is very impressive, and validates the use of this methodology.

\section{A probable interpretation}

We return to consider the interpretation of these observations. In the work of Overbury et al. (2), the effect of particle size on rate was presented as bilogarithmic plots for each of the two temperatures ( 273 and $298 \mathrm{~K}$ ), the slopes of the best lines through the sets of points for each catalyst being somewhat different. This gave rise to considerable discussion. Plots of this type, including the very familiar one that uses catalysts containing various supports (11), are predicated on the assumption that there is a continuous monotonic variation of activity with size. This assumption can then be explained by any of the physical characteristics that also vary uniformly, such as the fractions of edge and corner atoms, the length of the particles' perimeters, the melting temperature, or any combination of them. The results shown in Figure 3 indicate that activity depends on some property of the totality of the gold atoms in a particle; they therefore require another sort of explanation.

Examples of multiple 'Compensation' lines are comparatively common in the literature of heterogeneous catalysis $(4,5)$. They are particularly seen in the metal-catalysed reactions of hydrocarbons (3), where they have been used diagnostically (13), and with reactions on bimetallic catalysts $(3,4)$. It has been suggested (4) that catalyst systems adhering to a single 'Compensation' line share a common mechanism, or more precisely that they employ transition states of the same composition and architecture, but differing in energy. The cause of variation in $\mathrm{E}_{\text {app }}$ along a 'Compensation' line has been attributed to differences in the heats of adsorption, but unfortunately there is insufficiently extensive information for gold-catalysed CO oxidation to pursue the matter further at present. Evaluation of $E_{\text {true }}$ and of heats of adsorption necessitates measurement of 'orders of reaction' at several temperatures (3), and only the work of Bollinger and Vannice (14) does this, and that on a catalyst of rather poor dispersion. It is however a clear expectation that the kinetics on well and poorly dispersed catalysts will differ.

Finally we need to speculate on the cause of the twin 'Compensation' lines, and to identify the relevant factor that suffers a step change at particle sizes between about 2 and $4 \mathrm{~nm}$. The obvious factor is the metal to non-metal transition; this occurs when the size of the particle is so small that electron bands cannot from, so that it partakes of the character of a giant molecule (15); the exact size at which this occurs is not firmly established, but it probably happens in the size range 200-400 atoms, corresponding to about 2-3 nm. Failure to recognise that there may be an abrupt increase in activity as particle size decreases due to this cause, rather than gradual improvement caused by many of the more continuously changing features, may result from most catalysts having a distribution of particle sizes, sometimes very broad, of which only the lower tail is in fact the primary source of the activity. Assessment of size by a method such as XAFS that affords only a mean value, rather than by TEM which provides a distribution, is therefore not a reliable way of acquiring information that directly bears on activity.

The suggestion that high activity for $\mathrm{CO}$ oxidation is an attribute of the non-metallic state obtains support from recent proposals that relate it to bilayers of gold atoms $(16,17)$, that is, particles having extension in two dimensions, but being only two atoms thick. Whether such thin pancake-like particles are in fact non-metallic is a matter for future experimental and theoretical endeavour. This conclusion does not however eliminate the possibility that atoms of low coordination number are particularly adept at chemisorption, or that peripheral sites are not of importance.

Lest it be thought that the phenomenon of 'Compensation' in gold catalysis is the product of an over-heated imagination, the reader is referred to work on the decomposition of $\mathrm{H}_{2} \mathrm{O}_{2}$ catalysed by Pd-Au alloy wires (18); the kinetic parameters show excellent 'Compensation' with activation energies from 8 to $92 \mathrm{~kJ} \mathrm{~mol}^{-1}$. The range of $\mathrm{E}_{\mathrm{app}}$ values covered by variation of alloy composition and pre-treatment, and the proximity of all points to the line, makes this one of the most impressive 'Compensation' plots in the literature. 


\section{Conclusions}

When as in the case of the work described in reference 2 we have a variation in activation energy, there is no temperature at which a unique dependence of size on activity can be obtained; the activity profile at every temperature will differ. It is therefore necessary to use a 'Compensation' plot, which is a device for locating the activity of a catalyst for a given reaction relative to that of others, irrespective of the temperature at which it is measured. The change of $E_{a p p}$ along a 'Compensation' line is caused by variation of inputs form the heats of adsorption of the reactants. Duplicate 'Compensation' lines betoken different mechanisms, and the clear separation of the kinetic parameters for $\mathrm{CO}$ oxidation for catalysts containing large and small gold particles immediately suggests that in the more active catalysts most of the particles are non-metallic, while in the less active catalysts they are metallic. The cause of the variation of $E_{\text {app }}$ cannot be resolved until orders of reaction at several temperatures are available.

\section{About the author}

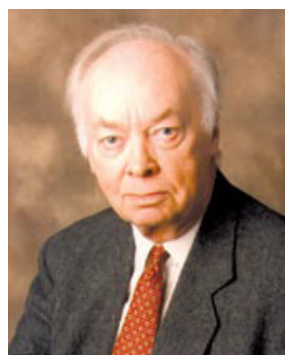

Geoffrey Bond held academic posts at Leeds and Hull Universities before joining Johnson Matthey plc in 1962 as Head of Catalysis Research. In 1970 he was appointed Professor in Brunel University Chemistry Department, and is now an Emeritus Professor at that University.

\section{References}

1 G. Bond and D. Thompson, Gold Bull. 2009, 42, 247

2 S.H. Overbury, V. Schwartz, D.R. Mullins, W.F. Yan and S. Dai, J. Catal. 2006, 241, 56

3 G.C. Bond, Metal-Catalysed Reactions of Hydrocarbons, Springer, New York, 2005, Ch. 5

4 G.C. Bond, Zeit. Physikal. Chem. NF, 1985,144, 21

5 G.C. Bond, M.A. Keane, H. Kral and J.A. Lercher, Catal. Rev.-Sci. Eng. 2000, 42, 323

6 F. Moreau, G.C. Bond and A.O. Taylor, J. Catal. 2005, 231, 105

7 F. Moreau and G.C. Bond, Appl. Catal. A: Gen. 2006, 302, 110

8 F. Moreau and G.C. Bond, Catal. Today, 2006, 114, 362

9 G.C. Bond, F. Moreau, R. Hughes, J.A. Moulijn, M. Makkee, K. Krishna and B.A.A. Silberova, Gold Bull. 2007, 40, 1

10 Y. Yuan, K. Asakura, H. Wan, K. Tsai and Y. Iwasawa, Catal. Lett. 1996, 42, 15

11 N. Lopez, T.V.W. Janssens, B.S. Clausen, Y. Xu, M. Mavrikakis, T. Bligaard and J.K. Nørskov, J. Catal. 2004, 223, 232

12 C. Rossignol, S. Arrii, F. Morfin, L. Piccolo, V. Caps and JI-L. Rousset, J Catal. 2005, 230, 476

13 G.C. Bond, Appl. Catal. A: Gen. 1999, 49, 41

14 M.A. Bollinger and M.A. Vannice, Appl. Catal. B: Env. 1996, 8, 417

15 G.C. Bond, C. Louis and D.T. Thompson, Catalysis by Gold, IC Press, London, 2006, Ch. 3

16 A.A. Herzing, C.J. Kiely, A.F. Carley, P. Landon and G.J. Hutchings, Science, 2008, 321, 1331

17 M. Chen and D.W. Goodman, Chem. Soc. Rev. 2008, 37 , 1860

18 D.D. Eley and D.M. MacMahon, J. Coll. Interface Sci.,1972, 38, 502 